\title{
A Marine Growth Detection System for Underwater Gliders
}

\author{
Enrico Anderlini ${ }^{\circledR}$, Daniel Alcaraz Real-Arce, Tania Morales ${ }^{\circledR}$, Carlos Barrera, José Joaquín Hernández-Brito (), \\ Alexander B. Phillips ${ }^{(0)}$, and Giles Thomas (i)
}

\begin{abstract}
Marine growth has been observed to cause a drop in the horizontal and vertical velocities of underwater gliders, thus making them unresponsive and needing immediate recovery. Currently, no strategies exist to correctly identify the onset of marine growth for gliders and only limited data sets of biofouled hulls exist. Here, a field test has been conducted to first investigate the impact of marine growth on the dynamics and power consumption of underwater gliders and then design an anomaly detection system for high levels of biofouling. A Slocum glider was deployed first for eight days with drag stimulators to imitate severe biofouling; then, the vehicle was redeployed with no additions to the hull for further 20 days. The mimicked biofouling caused a speed reduction due to a significant increase in drag. Additionally, the lower speed causes the steady-state flight stage to last longer and the rudder to become less responsive; hence, marine growth results in a shortening of deployment duration through an increase in power consumption. As actual biofouling due to $p$. pollicipes occurred during the second deployment, it is possible to develop and test a system that successfully detects and identifies high levels of marine growth on the glider, blending model- and data-based solutions using steady-state flight data. The system will greatly help pilots replan missions to safely recover the vehicle if significant biofouling is detected.
\end{abstract}

Index Terms-Biofouling, condition monitoring, fault detection, marine growth, underwater glider (UG).

\section{INTRODUCTION}

$\mathbf{U}$ NDERWATER gliders (UGs) are a type of autonomous underwater vehicle (AUV), which are designed for longterm observation of key physical oceanographic parameters [1]. The vehicles profile vertically through the water by changing their buoyancy through a variable buoyancy device (VBD), with wings generating a forward-motion component. Their simple propulsion system, which consists of the VBD, pitch control,

Manuscript received July 21, 2020; revised October 27, 2020 and January 19, 2021; accepted March 9, 2021. This work was supported by the European Union's Horizon 2020 research and innovation program under Grant 731103. (Corresponding author: Enrico Anderlini.)

Associate Editor: K. Asakawa.

Enrico Anderlini and Giles Thomas are with the Department of Mechanical Engineering, University College London, London WC1E 7JE, U.K. (e-mail: E.Anderlini@ucl.ac.uk; giles.thomas@ucl.ac.uk).

Daniel Alcaraz Real-Arce, Tania Morales, Carlos Barrera, and José Joaquín Hernández-Brito are with the Oceanic Platform of the Canary Islands (PLOCAN), 35214 Las Palmas, Spain (e-mail: daniel. alcaraz@plocan.eu; tania.morales@plocan.eu; carlos.barrera@plocan.eu; joaquin.brito@plocan.eu).

Alexander B. Phillips is with the Marine Autonomous and Robotic Systems, National Oceanography Centre, Southampton SO14 3ZH, U.K. (e-mail: abp@noc.ac.uk).

Digital Object Identifier 10.1109/JOE.2021.3066373 and either roll control or a rudder, is highly efficient. Therefore, although they operate at low velocities $(\approx 0.3 \mathrm{~m} / \mathrm{s})$, the UGs can be deployed for several months at a time.

The long deployment duration, in addition to regular surfacing to send data and receive new commands by satellite, can result in significant marine growth problems [2]. This issue is particular pronounced in shallow, warm, or tropical waters. Implementing design solutions to reduce the impact of biofouling, e.g., the use of antifouling paints and filling between the hull sections; or operational solutions, e.g., reducing the amount of time the vehicle spends in waters shallower than $150 \mathrm{~m}$, has proven generally effective to reduce, although not fully avoid, the problem. Marine growth results in an increase in the mass and mass of the glider, and a significant drop in speed. Therefore, biofouling can represent a severe operational problem, with the vehicle possibly needing premature retrieval at sea.

While some researchers have investigated the impact of marine growth on glider performance from field measurements, to date, there have been no systems developed to expressly detect and track marine growth for AUVs in real time. For instance, Haldeman, et al. [2] detected a significant drop in the vertical velocity of the glider due to biofouling from a 4-month-long deployment in the South Atlantic. Marine growth was observed in [3] to cause an increase in the drag coefficient and of $1^{\circ}$ in the angle of attack for the same pitch command for a glider deployment in the Indian Ocean lasting 7 months. Other researchers have investigated the impact of marine growth on glider performance using numerical approaches only. For example, medium-to-severe biofouling levels could cause a decrease in the lift coefficient of up to $40 \%$ and an increase in the drag coefficient of $90 \%$ [4] on lifting bodies.

To achieve a fully functional marine growth detection and isolation system, it would need be either embedded within the vehicle's software or operated remotely with the decimated data sent by the vehicle ashore via satellite after every dive. A remote condition monitoring system seems most appropriate, since onboard computational power is limited and power consumption should be reduced to a minimum to lengthen the mission duration [5].

It is possible to differentiate condition monitoring strategies into model- and data-based diagnostics [6]. While the former use dynamic models of the physical system, the latter analyze the sensor data. Model-based methods are more suitable for condition monitoring of new systems with limited data, whereas datadriven approaches are more general and can improve accuracy 
significantly if large amounts of data are available. Hong et al. [7] summarized model-based condition monitoring solutions with a focus on applications to strongly nonlinear and coupled systems. Reviews for machine learning and deep learning diagnostics could be found in [6] and [8] with a focus on machinery, and in [9] with a focus on wind energy.

Specifically for AUVs, most studies on fault detection and isolation focus on thrusters, inclusive of model-based solutions [10], radial basis function networks [11], Gaussian particle filter [12], and artificial immune system [13]. Harris [14] applied clustering solutions to identify a greater range of faults with unsupervised learning. Raanan et al. [15]-[17] introduced an automatic fault-detection system for long-range AUVs similar to UGs based on Bayesian nonparametric topic modeling techniques. The nearest neighbor classifier was found to be especially highly accurate over two different test sets. In [18] and [19], model-based methods were used to detect the loss of a wing and high levels of marine growth specifically for UGs. Here, the second study is extended with the design of data-driven solutions for the detection and isolation of marine growth on UGs.

This article describes field tests run as part of an EU Marine Robots Transnational Access (TNA) project to investigate the change in the dynamics of a Slocum G2 UG under simulated high levels of marine growth. In [2], it was clear that biofouling due to $p$. pollicipes could be severe and was represented by the growth of the shellfish along the junctions between different hull sections on a Slocum UG. Hence, marine growth will be simulated for the first time through rings with model molluscs attached to them, in a similar way to studs drag stimulators being used to induced turbulent flow in model ship scaled resistance tests [20]. As a result, the impact of biofouling on the dynamics, rudder performance, power consumption, and cost of transport (COT) of UGs will be investigated for the first time.

The data from the field test are then used to develop a new system that helps pilots detect and track the onset of marine growth. The tool merges the response of the UGs dynamic model in the vertical plane under steady-state conditions and an alternative data-based solution. As the collected data are limited to only two deployments of UG lasting less than one month combined, ensembles of regression trees are preferred over deep learning solutions due to their higher performance with small data sets [21]. Random forests have successfully been used for fault detection of steady-state faults [22]. Here, ensemble of regression trees are used for the first time to detect the onset of high levels of marine growth on UGs by tracking the residual of the predicted vertical velocity of the vehicle in the water. The residuals are used as input to a nearest neighbors clustering algorithm in addition to the AUVs drag coefficient estimated by a dynamic model to introduce a novel method for the detection, isolation, and identification of biofouling on UGs. The system is validated during a deployment presenting natural marine growth.

In Section II, the field test is described. Then, the marine growth detection and isolation system are developed. Finally, results are used to discuss the method's performance and its implications for UG operations.

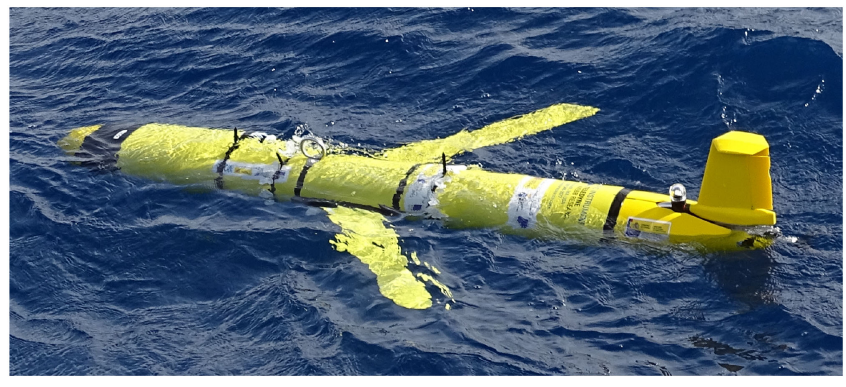

Fig. 1. Slocum G2 operated by PLOCAN.

\section{MARINE GROWTH FIELD TEST}

\section{A. Deployment Description}

Slocum is a type of UG manufactured by Teledyne Webb Research that has been in operation since the late 1990s [23], [24]. In this field test, the Slocum G2 UG operated by PLOCAN, as shown in Fig. 1, was employed (unit 492). The main actuator is the VBD (see Fig. 2), which consists of an oil bladder that can be extended or retracted from the pressure hull. When the bladder is outside the pressure hull, the vehicle's displacement and, thus, its net buoyancy increase and vice versa. Pitch is controlled by shifting the position of one movable battery pack with a dedicated mechanism. Yaw is controlled through a rudder, which is magnetically coupled to a servomotor to avoid an opening in the pressure hull.

As can be seen in Fig. 2, Slocums can perform multiple "yos" per dive (two in this particular case), i.e., the vehicle can sample the water column multiple times before returning to the surface to send and receive data by satellite and get a new position fix [25]. In particular, the minimum depth that the glider reaches between yos can be set to be lower than the surface to avoid marine traffic and reduce biofouling [2]. The data sent ashore need to be decimated to reduce the time that the vehicle spends on the surface, the power expenditure, and the actual financial cost associated with the transmission of the data by satellite (specifically, via Iridium). The data usually include the vehicle's orientation, its depth (from which the vertical velocity can be obtained), the actuator's signals, the capacity and voltage of the battery, the estimated location, samples of the scientific data of interest, and warnings from onboard health monitoring systems.

The data signals of interest from this field test are shown in Table I, in addition to the respective raw and decimated sample rates. Note that Slocums have two onboard central processing units (CPU): the navigation and scientific computers. The water conductivity, pressure, and temperature come from the scientific CPU, while all other variables from the navigation CPU. The navigation $\mathrm{CPU}$ is also connected to a pressure sensor that is used to estimate the vehicle's depth. In this field test, the only scientific sensor connected to the UGs scientific bay is the Seabird Scientific CTD sensor.

Example time series data for a typical dive cycle can be seen in Fig. 3. The vertical position of the vehicle in the water column (positive upward) is indicated by $z$, which is measured by an 


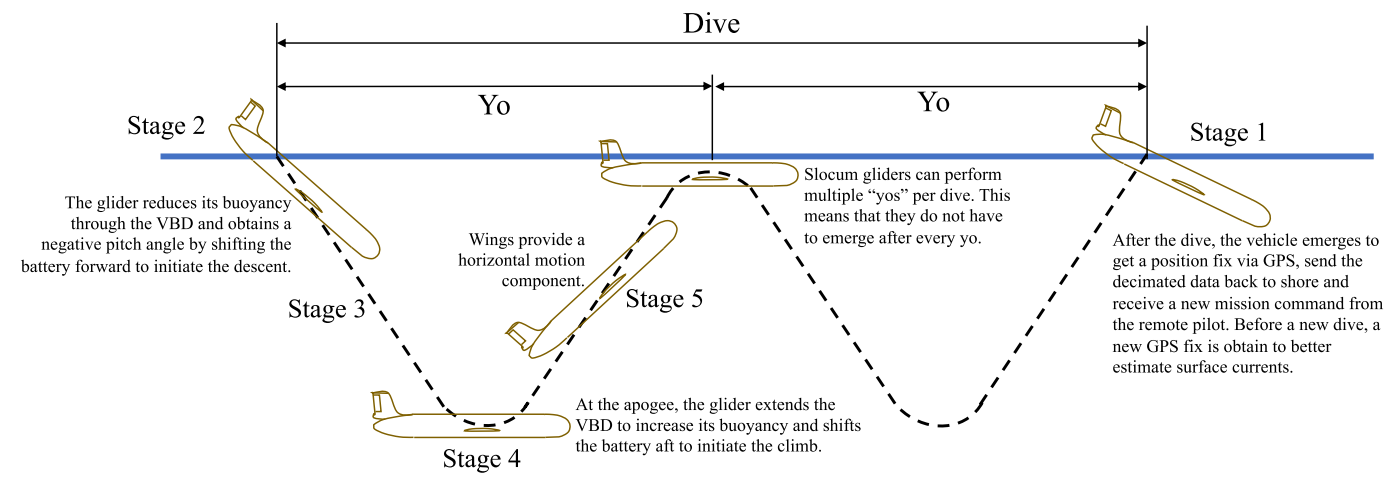

Fig. 2. Concept of operation of the UG. The drawing is not to scale: The vehicle operated in depths up to $1000 \mathrm{~m}$ deep and with the pitch angle ranging from $15^{\circ}$ to $30^{\circ}$.

TABLE I

SAMPLE RATES OF THE DATA SignALS OF INTEREST

\begin{tabular}{l|cc}
\hline Signal & Raw $[\mathbf{s}]$ & Decimated [s] \\
\hline Battery capacity & 60 & 60 \\
Battery position & 5 & 180 \\
Battery voltage & 60 & 60 \\
Depth & 5 & 15 \\
Heading & 5 & 30 \\
Pitch & 5 & 30 \\
Rudder angle & 5 & 60 \\
VBD volume & 5 & 180 \\
Water conductivity & 5 & 24 \\
Water pressure (navigation) & 5 & 15 \\
Water pressure (scientific) & 5 & 24 \\
Water temperature & 5 & 24 \\
\hline
\end{tabular}

onboard pressure sensor. Its time-derivative yields $\dot{z}$, the vertical velocity. The actuators' control signals are the volume of the VBD $V_{\mathrm{vbd}}$, the position of the moving battery pack $x_{\mathrm{b}}$, and the rudder angle $\delta_{\mathrm{r}}$. The roll $\phi$ and pitch $\theta$ angles are measured by tilt sensors, whereas a compass indicates the heading angle $\psi$. Here, the difference of the instantaneous yaw angle and the mean yaw angle over the whole dive $\bar{\psi}$ is used to favor the body-fixed over the inertial reference frame.

The field test actually comprised two separate deployments run with the same vehicle sequentially off the coast of Gran Canaria, as shown in Fig. 4. During the first deployment, the UG was deployed from February 17 to 26, 2020, off the east coast of the island after being covered with drag stimulators to mimic high levels of marine growth. In this test, the maximum depth was capped at $200 \mathrm{~m}$ to avoid possible structural and/or hydrostatic problems with the stimulators.

On February 26, the vehicle was recovered, the drag stimulators removed, and then the vehicle redeployed until March 17, 2020. During this deployment, the depth was increased to $1000 \mathrm{~m}$. While the scope of the first deployment was to provide a data set of a heavily biofouled glider, the second deployment had a scientific goal. By sharing the two sets, it has been possible to have data for the same glider with both a clean and a biofouled hull.

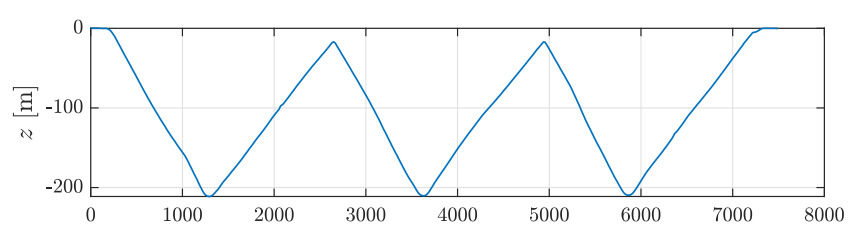

(a)

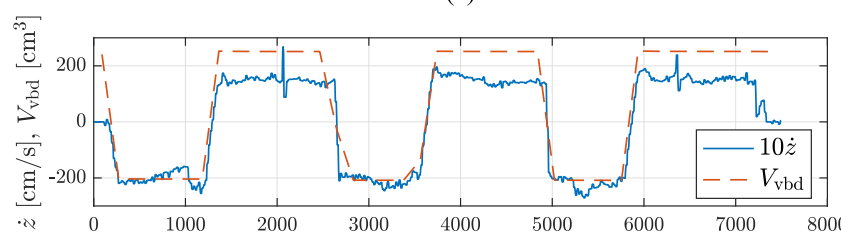

(b)

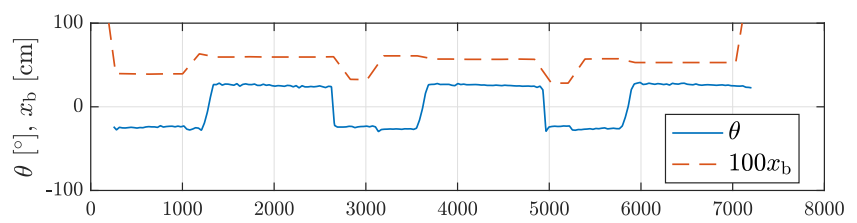

(c)

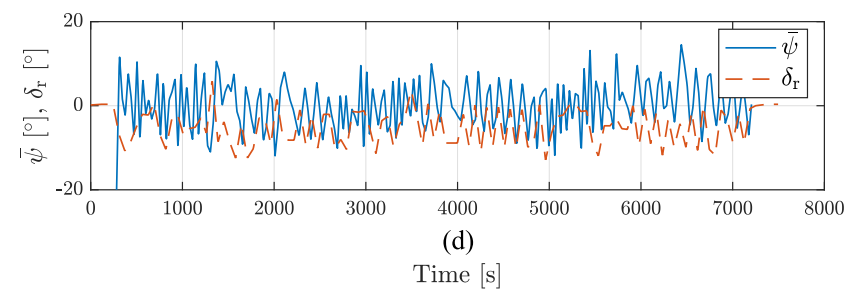

Fig. 3. Variation with time of the (a) vertical position, (b) vertical velocity and VBD volume, (c) pitch angle and battery position, and (d) yaw and rudder angles for a typical dive profile with three yos.

After the first few test dives with a single yo, the number of yos per dive was set to three during both deployments, as shown in Fig. 3. Both missions were constrained by time rather than battery energy. Hence, the full VBD capacity has been used to increase the UGs speed in the water at the expense of the higher energy cost. Thus, the magnitudes of the VBD volume and pitch angle were set to $260 \mathrm{~cm}^{3}$ and $26^{\circ}$, respectively, for most dives. To increase the range of the vertical velocity and pitch angle, a few dives were run with combinations of a VBD 


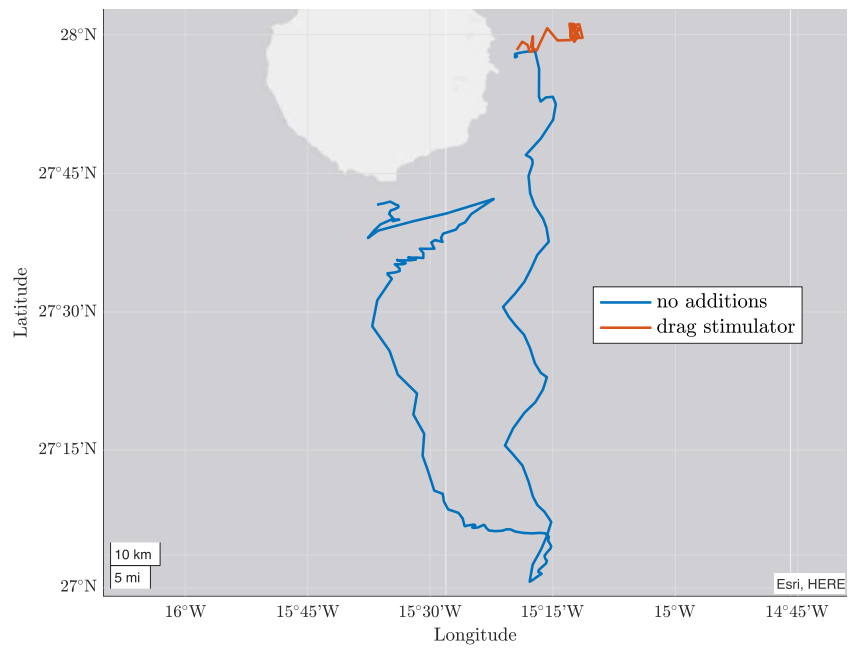

Fig. 4. Map of the two deployments.

volume of $-200 \mathrm{~cm}^{3}$ and $-260 \mathrm{~cm}^{3}$ in descents and pitch angles with a magnitude of $18^{\circ}, 26^{\circ}$, and $30^{\circ}$. In ascents, the full VBD capacity of $+260 \mathrm{~cm}^{3}$ was used at all times because of a perceived potential risk that the drag stimulators would compress with depth, thus contributing to a loss of net buoyancy.

\section{B. Drag Stimulators}

As described in [2], some of the most common occurrences of severe biofouling on UGs consist of the growth of shellfish (in particular p. pollicipes) on the nose and at the edges of the cylindrical sections of the hull. While the area around the nose presents lower flow speed due to the higher pressure associated with the front stagnation point, the junction of the cylindrical hull sections offers good grasping points for the roots of the shellfish larvae. Shallow dives, i.e., with a depth shallower than $200 \mathrm{~m}$, in warm waters have been found to especially contribute to marine growth. The main indicator of marine growth is a drop in the UGs vertical velocity for the same VBD volume and pitch angle settings.

In this study, artificial drag stimulators were designed and built to simulate high levels of marine growth as observed in [2]. In particular, a concept similar to drag stimulators to induce turbulent flow in ship model testing was envisaged [20]. Biofouling is expected to mostly impact the drag and net buoyancy of the vehicle. However, the change in net buoyancy is difficult to quantify and the UG had to be redeployed straight after the removal of the drag stimulators. Hence, to avoid serious ballasting challenges associated with two vehicle configurations, the drag stimulators have been designed to be approximately neutrally buoyant.

The final design of the drag stimulators can be seen in Fig. 5, as highlighted by the red boxes. In total, three drag stimulator belts were connected to the UG, each consisting of the following.

1) One 780-mm $\times 9$-mm nylon cable tie.

2) Heat shrink covers to increase friction with the hull and prevents the ties from creeping along the hull in water; the

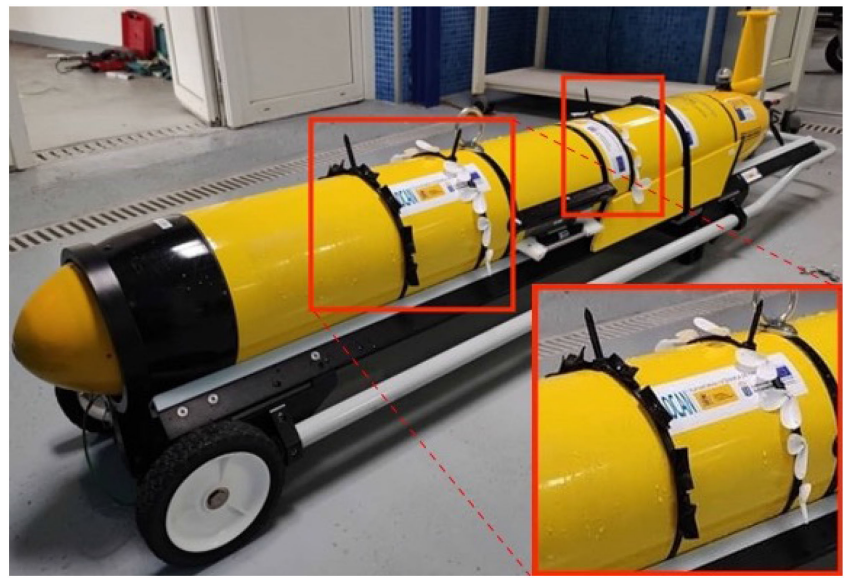

Fig. 5. Drag stimulators (highlighted by red boxes) added to the Slocum UG to simulate extreme levels of marine growth.

TABLE II

Mass, Displaced Volume, AND ORIENTATION OF THE DRAG STIMULators

\begin{tabular}{cccc}
\hline drag stimulator & mass $[\mathrm{g}]$ & volume $\left[\mathbf{c m}^{3}\right]$ & orientation \\
\hline Front & $52.39 \pm 0.5$ & $40 \pm 10$ & back-facing \\
Middle & $33.53 \pm 0.5$ & $25 \pm 10$ & forward-facing \\
Aft & $33.67 \pm 0.5$ & $25 \pm 10$ & back-facing \\
\hline
\end{tabular}

heat shrink is split into three parts to avoid contact with the two frames of the UGs trolley.

3) Ten 3-D-printed 40-mm-long mussels, each of which is connected to the cable tie at $50-\mathrm{mm}$ intervals through high strength fishing line passing through two drilled small holes. The front drag stimulator presents ten folded, 40-mm-wide neoprene strips instead of the 3-D-printed shells.

Although 3-D-printed parts have been used successfully in very deep waters [26], little information could be found on the use of parts built with selective laser sintering using Polyamide 12. Hence, dives with drag stimulators were capped to a maximum depth of $200 \mathrm{~m}$ for precaution.

Table II shows the total mass and displaced volume of each drag stimulator. As can be seen, the precision of the volume measurement is much lower than for the mass measurement. However, the drag stimulators are close to neutrally buoyant in the expected water density of the deployment site (approximately $1.027 \mathrm{~g} / \mathrm{cm}^{3}$ ).

During transport of the UG to the deployment site, one 3-Dprinted shell was lost from the middle drag stimulator. Hence, during the field test, the middle belt had only nine shells.

\section{Steady-State Dynamic Model}

The free-body diagram of the equilibrium condition for the steady-state flight is shown in Fig. 6(a) and (b) for descents and ascents, respectively. $B$ indicates the net buoyancy, $L$ the lift, and $D$ the drag force. $U$ is the surge velocity component in the body-fixed frame, $\theta$ the pitch, $\alpha$ the attack, and $\beta$ the glide-path angles. The glide-path angle indicates the angle of the flight path 


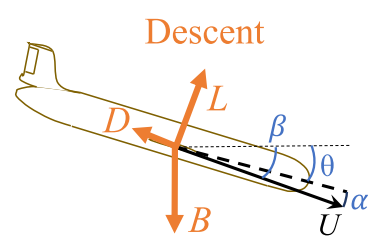

(a)

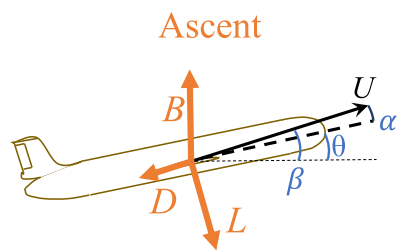

(b)

Fig. 6. Free-body diagram showing the forces acting on the UG in the vertical plane under steady-state conditions.

in the inertial reference system and is obtained from the sum of the pitch and attack angles: $\beta=\theta+\alpha$.

In both descents and ascents, the balance of forces yields [27], [28]

$$
B-L \cos \beta-D \sin \beta=0 \text {. }
$$

According to the standard dynamic model for Slocum UGs under steady-state conditions [28], the drag and lift forces can be modeled as

$$
\begin{aligned}
L & =\frac{1}{2} k_{\mathrm{L}} \alpha \rho S U^{2} \\
D & =\frac{1}{2}\left(k_{\mathrm{D}, 0}+k_{\mathrm{D}, \mathrm{L}} \alpha^{2}\right) \rho S U^{2}
\end{aligned}
$$

where $\rho$ is the water density, $S$ is the wetted surface area, $k_{\mathrm{L}}$, $k_{\mathrm{D}, 0}$, and $k_{\mathrm{D}, \mathrm{L}}$ are constants used to compute the lift and drag profile and induced drag. The water density is obtained from the water pressure, salinity, and temperature using the Gibbs Seawater Toolbox [29]. These properties are measured by the CTD sensor onboard the glider.

The net buoyancy force can be computed as

$$
B=g\left\{-m+\rho\left[V_{0}\left(1-\epsilon_{c} p+\alpha_{T}\left(T-T_{0}\right)\right)+V_{\mathrm{vbd}}\right]\right\}+\delta B
$$

where $g$ is the gravitational acceleration, $m$ is the UG mass, $V_{0}$ its reference volume, $\epsilon_{c}$ the absolute compressibility of the pressure hull, and $\alpha_{T}$ its thermal expansivity, with the reference temperature $T_{0}$. The values for the parameters can be found in Table III. $p$ is the water pressure and $T$ its temperature. The offset in the net buoyancy $\delta B$ is added over [28] to account for possible ballasting problems caused by the drag stimulators.

Substituting (2)-(4) into (1), it is possible to express the vertical velocity predicted by the model $\dot{z}_{\mathrm{m}}$ as follows [28]:

$$
\dot{z}_{\mathrm{m}}=\sqrt{U^{2}} \sin \beta
$$

where

$$
U^{2}=\frac{B \sin \beta}{0.5 \rho S\left(k_{\mathrm{D}, 0}+k_{\mathrm{D}, \mathrm{L}} \alpha^{2}\right)}
$$

TABLE III

SUMmary OF THE FIXED MODEl PARAMETERS

\begin{tabular}{cccc}
\hline parameter & value & units & source \\
\hline$g$ & 9.81 & {$\left[\mathrm{~m} / \mathrm{s}^{2}\right]$} & {$[28]$} \\
$S$ & 0.1 & {$\left[\mathrm{~m}^{2}\right]$} & {$[28]$} \\
$\epsilon_{c}$ & $6.4 \times 10^{-6}$ & {$\left[\mathrm{dbar}^{-1}\right]$} & manufacturer \\
$\alpha_{T}$ & $5.3 \times 10^{-5}$ & {$\left[{ }^{\circ} \mathrm{C}^{-1}\right]$} & manufacturer \\
$m$ & 58.2428 & {$[\mathrm{~kg}]$} & ballasting \\
$V_{0}$ & 56,730 & {$\left[\mathrm{~cm}^{3}\right]$} & ballasting \\
$T_{0}$ & 19.43 & {$\left[{ }^{\circ} \mathrm{C}\right]$} & ballasting \\
\hline
\end{tabular}

$$
\alpha=\frac{k_{\mathrm{D}, 0}+k_{\mathrm{D}, \mathrm{L}} \alpha^{2}}{k_{\mathrm{L}} \tan (\beta)} .
$$

Equation (7) requires an iterative solution. Additionally, an optimization needs to be run to find the hydrodynamic parameters specific to the UG. The cost function is expressed as

$$
J=\left[\frac{1}{I} \sum_{i=1}^{I}\left(\dot{z}_{i}-\dot{z}_{\mathrm{m}, i}\right)^{2} \forall i \in \mathbb{R}\right]+\left[\sum_{j=1}^{J} j \forall j \notin \mathbb{R}\right] .
$$

In (8), $i$ indicate all points for which a numeric cost value is obtained, so that $\dot{z}_{i}$ is the actual and $\dot{z}_{\mathrm{m}, i}$ the modeled vertical velocity of the UG for the $i$ th point. $j$ indicates all points for which $U^{2}<0$. Imposing an additional cost on the number of nonnumeric values speeds up convergence as compared with that in the work by Merckelbach et al. [28].

Here, a global search optimization is run to find the desired parameters for each deployment: $k_{L}, k_{D, 0}, k_{D, L}$, and $\delta B$. A scatter search [30] is used to generate trial points within the search space from which a constrained, nonlinear programming solution is found based on a trust region method based on interior point techniques [31]. The scatter search algorithm then assesses the cost function of the solutions to update the trial points and continue the minimization until convergence onto the global optimum. The MATLAB GlobalSearch and fmincon tools were used in the practical implementation, with default settings. The lift, drag, induced drag, and buoyancy offset values were constrained to $k_{L} \in[0,25] \mathrm{rad}^{-1}, k_{D, 0} \in[0,0.4]$, $k_{D, L} \in[0,12] \mathrm{rad}^{-2}$, and $\delta B \in[-3,3] \mathrm{N}$, respectively.

\section{Power-Consumption Analysis}

The impact of marine growth on the power consumption of the UG can also be analyzed. In general, it is possible to identify five main operational stages that will contribute to different power consumption profiles, which are shown in Fig. 2.

Stage 1) Communications. When the UG is on the surface before and after each dive, satellite communications drive the power consumption.

Stage 2) Surface-actuation. As shown in Fig. 3, there is an actuation stage during which the VBD is retracted and the battery pack is shifted forward to initiate a descent.

Stage 3) Descent-steady-state conditions. During descent, the UG dives under steady-state conditions.

Stage 4) Apogee-actuation. At the apogee, the VBD is extended and the battery pack is shifted aft to initiate the ascent. 


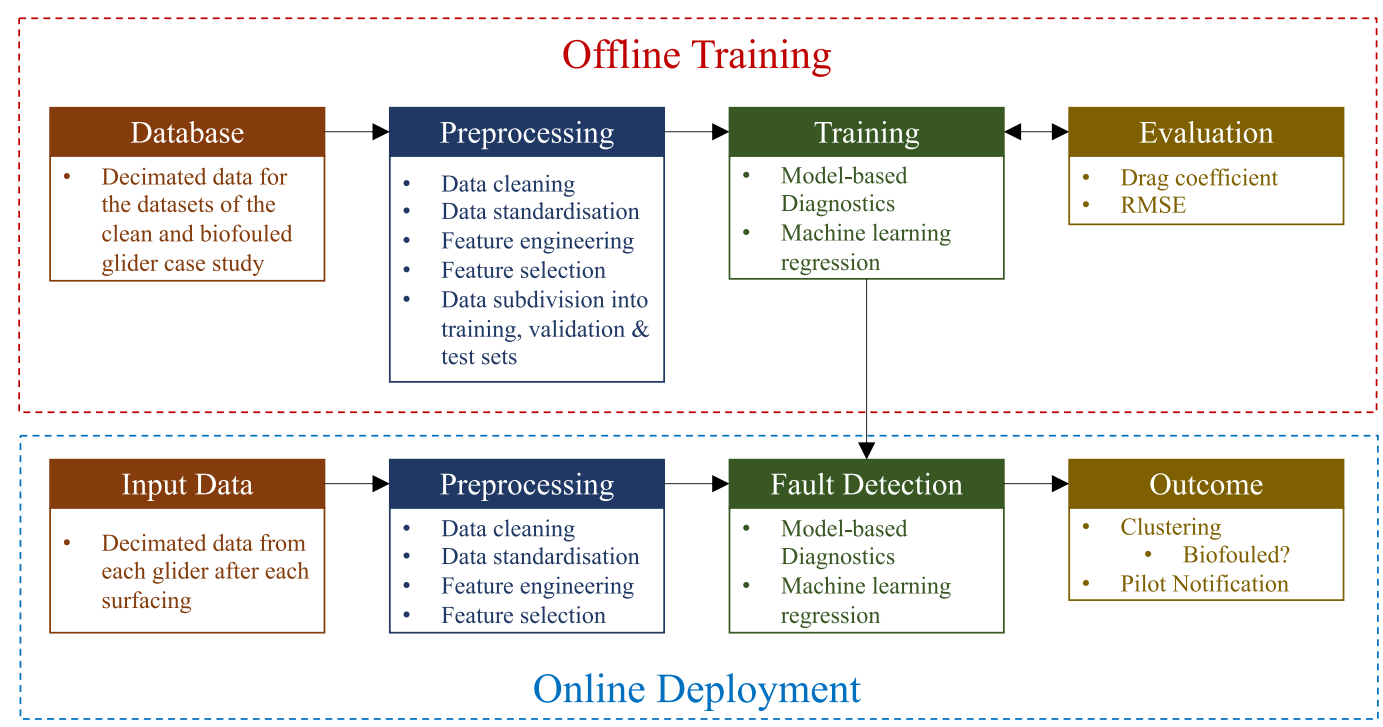

Fig. 7. Diagram of the new marine detection system.

Stage 5) Ascent—-steady-state conditions. During ascent, the UG climbs under steady-state conditions.

The last four stages will repeat for every yo in a dive.

The data are cleaned to obtain the energy consumption (from the signal of the battery capacity and voltage) and duration of each stage for all dives and yos. As Stage 1 (communications) happens only once per dive while the other ones once per yo, the energy consumption and duration of the first stage are repeated for all yos to express the data in a table format. One set of data is obtained for the clean hull and one for the severely biofouled hull. The energy consumption and duration of the telemetry stage (Stage 1) is a function of the amount of data that needs to be transmitted by satellite. The energy consumption and duration of the dynamic response stages (Stage 2 and Stage 4) are a function of the change in the actuators' settings. During the two deployments, the glider was operated at maximum VBD volume in both clean and biofouled conditions. Therefore, the most noticeable changes due to marine growth were expected under the steady-state operation (Stage 3 and Stage 5). Hence, the data from the steady-state descents and ascents were merged and subjected to further statistical analysis. Since the maximum and minimum depths can have a large influence on the energy consumption and duration of each steady-state stage, only data from dives with similar maximum and minimum depths were considered (up to $200 \mathrm{~m}$ ).

First, an analysis of covariance (ANCOVA) [32] was run to determine whether there is a significant difference in the mean power consumption during steady-state conditions between the clean and biofouled hull (Stage 3 and Stage 5). The analysis also controls for the effect of the mean absolute VBD volume and pitch angle during either the ascent or descent by studying the dependence of the mean power consumption on these variables through linear regression. A significance level of $99 \%$ was used.

If there is a significant difference in the mean power consumption under steady-state conditions between clean and biofouled hulls, this is likely to be due to higher actuation levels, in particular, the pitch control and rudder mechanisms. Since marine growth is known to result in a lower UG velocity [2], the rudder will become less effective. Hence, the change in variance of the rudder angle between clean and biofouled conditions is also studied.

First, the Lilliefors test was run to test the null hypothesis that the rudder angle during steady-state conditions is normally distributed to a significance level of $99 \%$ for both sets [32]. If the data are not normally distributed, then Levene's quadratic test was used to check whether the two data sets present different variance to a significance level of $99 \%$.

\section{MARINE GROWTH DETECTION}

A novel system was developed to detect and isolate marine growth on UGs, as can be seen in Fig. 7. The tool was created and tested offline using the data collected during the field test for a Slocum G2. After successful testing, the trained fault detection and isolation system can be applied online to notify pilots of possible high levels of marine growth on the UG after each surfacing and satellite connection, e.g., through an over-the-horizon command and control infrastructure, as described in [33].

For both offline training and online deployment, decimated data sent remotely will be analyzed, so that the preprocessing stages are identical. To improve the accuracy and robustness of the system, both model- and data-based algorithms are used to detect marine growth. A clustering algorithm is then used to classify the UGs behavior as either normal or severely biofouled using the drag coefficient estimated by the dynamic model and the root-mean-square error (RMSE) of the machine learning regression as input. The automated classification step reduces reliance on rule-based solutions and their poor generalization.

Model-based diagnostics use a model constructed from indepth knowledge of the system dynamics and can be, thus, applied on newly developed systems [5]. Conversely, data-based solutions can be applied only after sufficient training data are 
available but can offer significant improvements in condition monitoring accuracy [34]. The challenge for a data-based approach is the small size of the data set obtained in the field test, which means that a machine learning strategy is preferable over deep learning [21].

\section{A. Preprocessing}

UGs spend most of their descents and ascents in steady-state flight. Hence, the complexity of the marine growth detection system can be greatly reduced by using only data corresponding to steady-state conditions. In this study, only decimated data sent by satellite were considered. The UG was programmed remotely at the start of the mission to ensure that the signals of interest are presented in the files sent remotely. Hence, the data stored in the .SBD and .TBD files for the navigation and scientific CPUs, respectively, were converted from binary to ASCII format using the Python dbdreader module. Afterward, the points were imported into the MATLAB environment.

All dives with less than 10 sample points of the variables of interest shown in Table I or with a maximum depth shallower than $25 \mathrm{~m}$ were removed to ignore the trial dives at either the start or end of each deployment. All signals were then resampled by linear interpolation for exactly the same time stamps, with a time step of $30 \mathrm{~s}$. The navigation and scientific computers were synced through the pressure signal, which is measured by both units.

The variables of interest, such as vertical velocity and water density, were computed from the raw signals.

To reduce the transient effects caused by operations on the surface and apogee, points within $10 \mathrm{~m}$ from the surface (or the highest point of the ascent) and the maximum depth were ignored. All points that present a nonnumeric value for any signals were removed. Due to the large time step, it was difficult to suitably identify dynamic effects during the descents and ascents. Hence, all points in the cleaned depth range were considered, which may cause some noise.

For the machine learning strategy, using the data values expressed in the physical units may result in bias toward values with much higher mean or standard deviation values, e.g., the VBD volume. Hence, for the data-based solutions, the input data were standardized [35]. Conversely, the dimensional units were used for the dynamic model.

Feature selection and engineering are specific to the machine learning approach and will, thus, be covered in Section III-C.

\section{B. Model-Based Diagnostics}

The model-based diagnostics rely on the dynamic model and global optimization described in Section II-C. However, the range of all signals is much more limited over each dive than over the whole deployment. Hence, the data regression may be biased. Therefore, the optimization problem is reduced to finding the drag coefficient $k_{D, 0}$ and net buoyancy offset $\delta B$ to halve the dimensionality of the problem. The values of the lift and induced drag coefficients are, thus, preset to values found in [28] and [27], respectively: $k_{\mathrm{L}}=7.5$ and $k_{\mathrm{D}, \mathrm{L}}=3$ for Slocum $\mathrm{G} 2 \mathrm{UGs}$. The optimization is then run for each dive with a moving window, after the new decimated data are received once the UG surfaces and establishes a connection. The drag coefficient can, then, be tracked to identify rising levels of marine growth.

\section{Data-Based Diagnostics}

Machine learning strategies are becoming increasingly popular for condition monitoring [6]. The error between the predicted and actual vertical velocity can be an effective anomaly indicator. Hence, the detection of high levels of marine growth on UGs can be simplified to estimating the vertical velocity in steady-state flight with a machine learning algorithm. If the method is trained with data from a UG with a clean hull, then the RMSE will increase with biofouling levels. Hence, a classification algorithm can then be used to differentiate conditions relating to a clean and to a heavily biofouled hull.

The data set from the field test is relatively small: The deployment with the drag stimulators presents 10135 points while the deployment with no additions to the hull 24130 points. Hence, machine learning algorithms are more suitable than deep learning [21]. Decision regression trees are a powerful prediction method, which exploit nonlinear relationships between inputs and outputs. Decision trees rely on partitioning the data along the predictor axes into subsets with homogeneous values of the dependent variable [36]. The data segmentation occurs through recursive binary splitting to reduce the residual sum of squares of the prediction error. The splitting is repeated at each decision node until a termination criterion is reached. The structure resulting from the splits resembles a tree, with branches and leaves. The predicted value is obtained from the mean value of training observations in the region to which the test value belongs.

Individual decision trees tend to overfit the training data. Hence, to improve generalization, the results from many regression trees can be aggregated to reduce the bias toward specific features of individual weak learners if their errors are uncorrelated [37]. In particular, there are two aggregation strategies: boosted and bootstrap-aggregated (or bagged) decision trees [38]. An ensemble of many weak learners results in a strong learner with higher prediction accuracy.

Boosting is an ensemble method where learners operate in series. Hence, the first regression trees fit the data and its error is fed to the following tree, which then fits the error from the previous tree, and so on. The weight associated with higher prediction errors is increased so that the ensemble model can refine the prediction with learning. The boosting method can be extended to gradient boosting by applying the gradient descent algorithm to optimize any differentiable loss function. Here, least-square boosting (LSBoost) is used, where the RMSE of the prediction is to be minimized. At every step, the ensemble fits a new tree to the difference between the observed response and the aggregated prediction of all trees grown previously.

Conversely, bagging is an ensemble method where the learners operate in parallel. Random samples with replacement are selected from the training set, and trees are fitted to these samples. The results from all individual trees are, then, averaged to predict the output values. The method is extended to random forests by randomly selecting predictors for each decision split. This 
decorrelates the regression trees and improves the robustness of the overall ensemble. Here, the number of predictors to select at random for each split is equal to one-third of the number of predictors.

While gradient boosting may result in greater precision, random forests are less prone to overfitting [38]. Hence, both methods are used in this article, using the MATLAB Machine Learning toolbox implementation.

The variable to be predicted for the marine growth detection is the vertical velocity for the steady-state conditions. To achieve the same result, the dynamic model relies on the VBD volume, the pitch angle, the battery position, the water density, pressure, and temperature. Therefore, the input features for the machine learning algorithms are $\theta, x_{\mathrm{b}}$ and the following two engineered features:

$$
\begin{aligned}
& f_{1}=\rho V_{\mathrm{vbd}} \\
& f_{2}=\rho V_{0}\left(1-\epsilon_{c} p+\alpha_{T}\left(T-T_{0}\right)\right) .
\end{aligned}
$$

The first engineered feature $f_{1}$ describes the change in displaced mass associated with the VBD, whereas the second feature $f_{2}$ is the change in displaced mass due to compressibility and thermal expansivity effects.

The importance of all features is determined from the outof-bag error of the random forest model. By selecting random samples with replacement, some observations are omitted for each decision tree [38]. For each observation, the out-of-bag prediction corresponds to the mean from all trees in the ensemble for which the observation is out of bag. Hence, the mean-square error of the out-of-bag predicted responses as compared with the actual observations of the training set can be used to produce an unbiased estimator of the actual error of the overall ensemble. From the out-of-bag error of the random forest model trained on the data with all features, only the pitch angle, battery position, and the product of the water density and VBD volume are used as features for the gradient boosting and random forest strategies. These values are standardized using the statistics of the training set before being used by the algorithms. The trained model is able to deal with data outside the training set range after it is deployed. However, if the data presents a different distribution, the UGs behavior is likely to be flagged as anomalous. The standardization reduces the impact of outliers as compared with normalization.

The LSBoost and random forest models are trained using the data from the first six days of the deployment of the UG with no additions, i.e., the data corresponding to a clean hull. Crossvalidation and the out-of-bag errors are used to determine the number of trees and minimum leaf size and reduce the risk of overfitting.

\section{Clustering Analysis}

To improve the robustness of the marine growth detection system to noise due to ocean currents and few points per dive in the decimated data, the results from the model- and databased diagnostics are merged. In particular, the drag coefficient and the RMSE from the random forest are used as input to a classification algorithm to reduce the specificity and bias of rulebased solutions. To generalize the data collected in the field test to other Slocum UGs, the drag coefficient and RMSE of the random forest are standardized with the $z$-score, i.e., centered to have a mean of 0 and standard deviation of 1 , using the statistics of the training set to reduce the importance of outliers.

The $k$-nearest neighbors (KNN) algorithm is used for the classification. The scheme is an instance-based learner that estimates the output values by observing the $k$ points closest to the input data in the search space, using a specified distance metric [38]. Here, the Mahalanobis distance is used. When the nearest points are found, a weighted sum of the $k$-nearest neighbors is taken to yield the output prediction.

KNN is easy to implement and can be applied for both supervised and unsupervised learning. In unsupervised learning, the algorithm is used to identify clusters in the data. Hence, it is highly suitable for the marine growth detection problem, since two distinct clusters are expected for the clean and severely biofouled UG. The normalization and standardization processes reduce the primary problem of the algorithm, namely its susceptibility to the scale of the input features, since it is a distance-based method. Although the KNN strategy has poor scalability to large problems, the small data set of the field test does not cause any issues. The MATLAB Machine Learning toolbox implementation is used in this study.

\section{E. Validation of the Anomaly Detection Algorithm}

As shown in Fig. 7, the marine growth detection system is subdivided into offline training and online deployment. Here, the data from the deployments is used for the validation of the algorithms with a number of steps.

First, an ensemble of bagged trees and an ensemble of boosted trees are trained with the data for the first six days of the second deployment, characterizing little to no biofouling conditions. In particular, the steady-state data are split into disjoint training and test subsets, each comprising $70 \%$ and $30 \%$ of the points, respectively. For bagging, the chosen architecture consisting of 25 trees with a minimum leaf size of five is selected to minimize the out-of-bag RMSE. For LSBoost, fivefold cross-validation is used for hyperparameter selection. The chosen architecture has 100 learning cycles and uses least-squares boosting.

The RMSE of the ensemble of bagged regression trees, the RMSE of the ensemble of boosted regression trees, and the drag coefficient are used as input to the KNN clustering algorithm. Here, the training set is increased to include the data for the simulated high level of biofouling with drag stimulators in addition to the data for the clean hull. Note that only the data points from the previously obtained set to test the regression methods (i.e., $30 \%$ of all the samples from the first six days of the second deployment) have been used in this second study in addition to all data points for the deployment with mimicked biofouling. The three input variables are thus standardized with respect to this data set. To verify the performance of the clustering algorithm, the data are split into disjoint training and test subsets, each comprising $70 \%$ and $30 \%$ of the points, respectively. Fivefold 

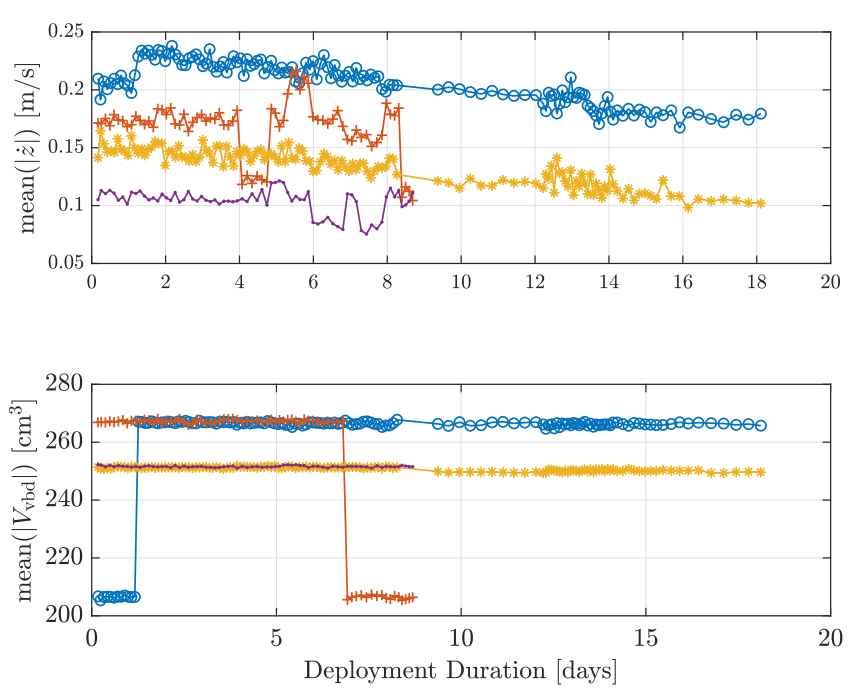

- - no additions - descents $\longleftarrow$ drag stimulators - descents
$-*$ no additions - ascents $\longleftarrow$ drag stimulators - ascents

Fig. 8. Variation with time of the (a) mean absolute vertical velocity and (b) VBD volume in ascents and descents for both deployments.

cross-validation is employed for the KNN classification algorithm.

The regression and classification methods are then applied to the data for the second deployment, which includes data for natural blossoming biofouling on the UG, to assess their generalization performance with unseen data. However, note that the data excludes the data belonging to the respective training sets.

\section{RESULTS AND DISCUSSION}

In this section, first, the difference in the response of the UG with a clean and a simulated severely biofouled hull is analyzed. Then, the fault detection system is deployed to identify and track marine growth during the run with the "clean" hull, i.e., with no drag stimulators, due to actual natural biofouling.

Hence, there are three types of data in the analyzed deployments: 1) clean hull with very little marine growth;2) simulated high levels of marine growth through drag stimulators; and 3) real biofouling, corresponding to the end of the second deployment. Note that in Section IV-A, the data for the severely biofouled hull correspond to the simulated high levels of biofouling with drag stimulators 2), while the data for actual biofouling 3) are used to validate the anomaly detection system in Section IV-B. The real marine growth data 3) can be used to update the comparison between the biofouled and clean hull in the future.

\section{A. Comparison Between Biofouled and Clean Glider}

1) Data Averaged Over Each Dive: The variation with deployment duration of the mean absolute vertical velocity, VBD volume, pitch angle, and battery position can be seen in Figs. 8 and 9. Note that there are some discrepancies in the output values for $V_{\mathrm{vbd}}$ and $\theta$ from the input settings due to the calibration of the

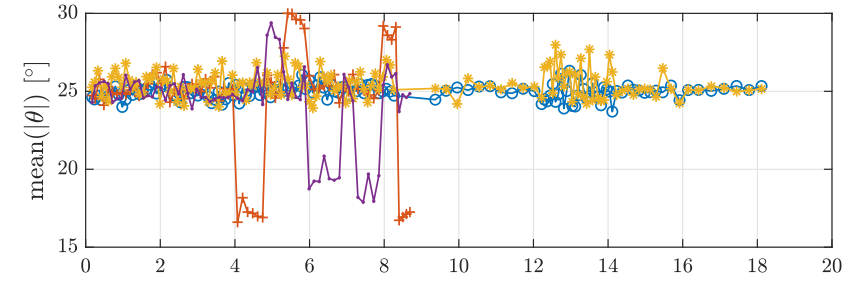

(a)

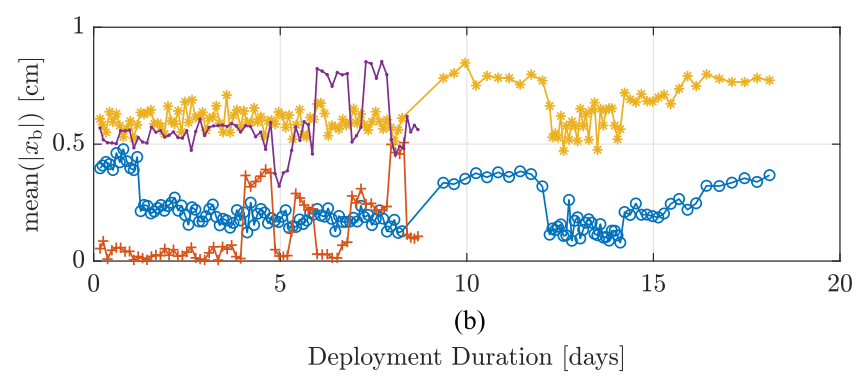

- no additions - descents $\multimap$ drag stimulators - descents
- no additions - ascents $\longrightarrow$ drag stimulators - ascents

Fig. 9. Variation with time of the (a) mean absolute pitch angle and (b) battery position in ascents and descents for both deployments.

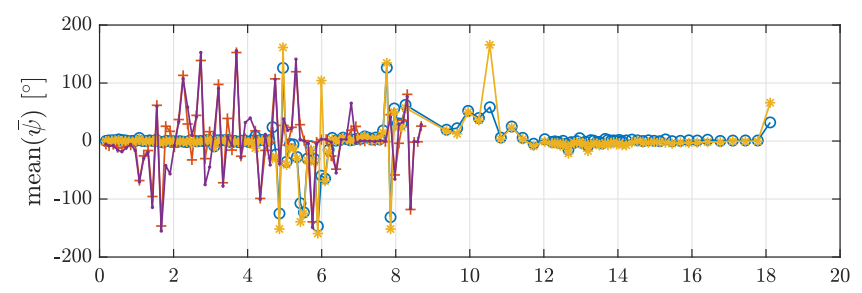

(a)

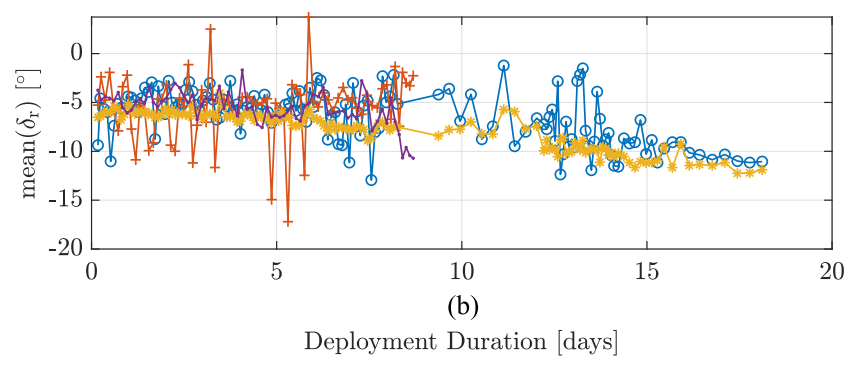

- no additions - descents — drag stimulators - descents
* no additions - ascents $\longleftarrow$ drag stimulators - ascents

Fig. 10. Variation with (a) time of the mean yaw and (b) rudder angles volume in ascents and descents for both deployments.

parameters of the control system on the glider. Fig. 10 shows the variation with deployment duration of the mean yaw and rudder angles. The data from the last two days of the deployment with no additions to the UGs hull are ignored, since points were stored only for descents to reduce data transmission costs while the vehicle was waiting on station for retrieval due to the Covid-19 outbreak.

From Fig. 8(b), it is clear that full VBD volume has been used for the ascents for safety reasons, while a lower volume 


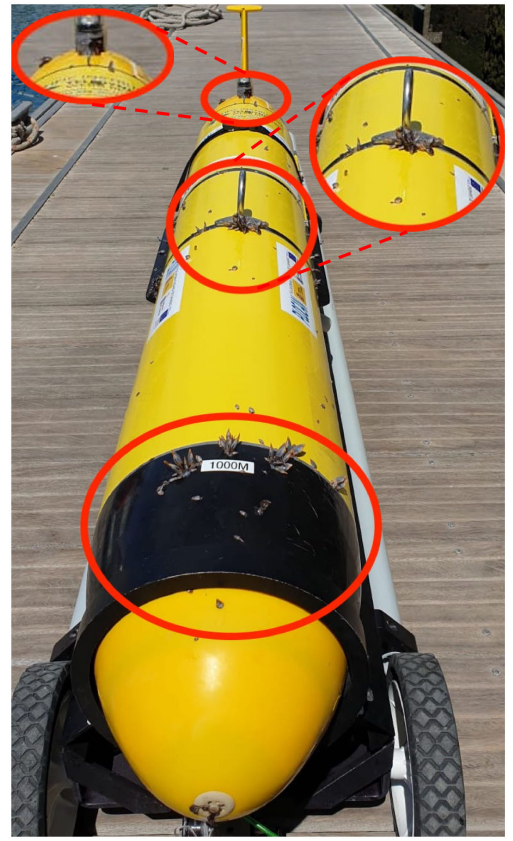

Fig. 11. Actual biofouling due to $p$. poliicipes on the UGs hull at the end of the field test.

of $200 \mathrm{~cm}^{3}$ was used for a few dives for both deployments to increase the value's range. Similarly, a few dives of the vehicle with drag stimulators have been run for different pitch angle settings, while the pitch angle range is more limited for the deployment with no additions to the UGs hull due to the purely scientific nature of the mission. The deliberate variation of the VBD volume and pitch angle control settings to increase their range resulted also in changes in the vertical velocity and pitch angle of the UG, as shown in Figs. 8 and 9. Less deliberate variation is visible for the second deployment because its primary goal was primarily scientific.

From Fig. 10, it is clear that the mean yaw and rudder angles present much greater variation for the deployment with the UG with drag stimulators, although the variance increased with mission duration for the deployment also for the UG with no additions. By looking at Fig. 8(a), the mean vertical velocity significantly decreases with time for the deployment with no additions. From [2], this is a sign of possible marine growth. Indeed, when the glider was recovered, the vehicle presented a significant extent of biofouling along its whole length, with strong concentrations at the bow, near the lifting support ring and the aft fin, as shown in Fig. 11. In particular, the culprit was found to be $p$. pollicipes like in [2]. Despite the relatively short overall mission time (one month), the initial dives in shallow, warm waters of the vehicle with the drag stimulators must have created perfect conditions for the molluscs to take root on the hull, while still being undetected during the first recovery of the vehicle after eight days. Even though the UG was run to a depth of $1000 \mathrm{~m}$ after approximately five days of the second deployment, the shellfish was already settled on the vehicle and could grow undisturbed. Therefore, only the data from the first six days of the second deployment (where the hull has no
TABLE IV

Parameters of the Dynamic Model for the Two Hull Conditions

\begin{tabular}{ccccc}
\hline Hull state & $k_{L}\left[\mathrm{rad}^{-1}\right]$ & $k_{D, 0}$ & $k_{D, L}\left[\mathrm{rad}^{-2}\right]$ & $\delta B[\mathrm{~N}]$ \\
\hline Clean & 2.659 & 0.140 & 4.759 & -0.537 \\
Biofouled & 3.881 & 0.269 & 6.525 & -0.521 \\
\hline
\end{tabular}

additions) are used for the comparison of the UGs response with a clean (no additions and noticeable biofouling from the mean vertical velocity plot) and severely biofouled hull (with the drag stimulators). The whole data set of the vehicle with no additions is then used for the development and testing of the marine growth detection system.

2) Dynamic Model Analysis: Table IV shows the parameters of the dynamic model found by the global optimizer for the UG with the clean (with no additions and no significant marine growth) and severely biofouled hulls (with drag stimulators). The results are in line with observations from many studies summarized in [3] except for the lift coefficient.

As can be seen in Table IV, the drag stimulators cause an increase of $92 \%$ in the drag coefficient and $37 \%$ in the induced drag coefficient. Conversely, there is no significant change in the net buoyancy offset thanks to the neutrally buoyant design of the drag stimulators, which has helped to isolate the impact of marine growth on the vehicle's drag. In fact, a small change in the buoyancy offset is expected for actual shellfish biofouling due to molluscs being slightly negatively buoyant.

From Table IV, the drag stimulators increase the lift coefficient by $46 \%$. This is mainly due to the extremely large size of the 3-D-printed shells of the drag stimulators. In fact, real biofouling may cause a reduction of lift coefficient [4]. It is also interesting to note that the global optimizer finds a value of the lift coefficient for the clean hull that is $65 \%$ smaller than the one observed by Merckelback et al. [27], [28] as obtained from an iterative solution (7.5).

The glide polar plot for the UG is shown in Fig. 12 using the parameters in Table IV for the full VBD volume of $260 \mathrm{~cm}^{3}$, a water density of $1.027 \mathrm{~kg} / \mathrm{m}^{3}$ and ignoring the compressibility and thermal expansivity of the hull. Due to the low ratio of the estimated lift and drag coefficients, the polar plot presents higher minimum glide angle and glide angle for maximum horizontal speed than theoretical predictions in [3] and [4]. The discrepancy is likely to be due to the limited range of the $\theta-\dot{z}$ search space in the analyzed deployments. As the variables' range is even more constrained on individual dives, reducing the search space for the system identification to two parameters for individual dives is justified.

3) Power-Consumption Analysis: From the ANCOVA, the mean power consumption in each descent or ascent has been found to be significantly different for the clean and biofouled hull data sets $(p=0.04 \%<1 \%)$. Additionally, the values are significantly affected by the mean VBD volume $(p \ll 1 \%)$ while the contribution of the pitch angle is not deemed significant to a $99 \%$ level $(p=2.04 \%)$. However, the ANCOVA results should be treated with caution, as it assumes linear relationships 


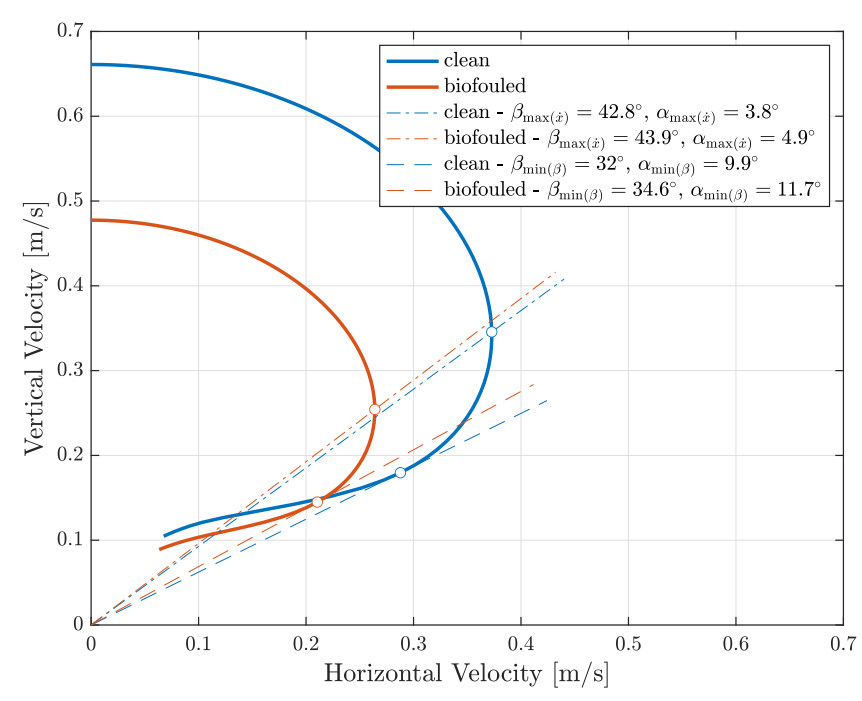

Fig. 12. Glide polar plot for the UG using the parameters in Table IV for the full VBD volume of $260 \mathrm{~cm}^{3}$, a water density of $1.027 \mathrm{~kg} / \mathrm{m}^{3}$, and ignoring the compressibility and thermal expansivity of the hull.

TABLE V

MEAN AND Standard DeViation of the UGs POWER CONSUMPTION DURING THE FIVE IDENTIFIED OPERATIONAL STAGES

\begin{tabular}{l|cc|cc}
\hline Operational phase & \multicolumn{3}{|c}{ Clean } & \multicolumn{3}{c}{ Biofouled } \\
& $\bar{P}[\mathrm{~W}]$ & $s_{P}[\mathrm{~W}]$ & $\bar{P}[\mathrm{~W}]$ & $s_{P}[\mathrm{~W}]$ \\
\hline communications & 4.688 & 0.164 & 4.788 & 0.157 \\
actuation - surface & 2.296 & 0.526 & 2.340 & 0.479 \\
steady-state descent & 1.875 & 0.094 & 2.012 & 0.198 \\
actuation - apogee & 25.961 & 1.311 & 25.549 & 1.399 \\
steady-state ascent & 1.742 & 0.018 & 1.742 & 0.028 \\
\hline mean & 7.312 & 0.423 & 7.286 & 0.452 \\
\hline
\end{tabular}

TABLE VI

MEAN AND STANDARd DEVIation OF THE DURATION OF THE Five IDENTIFIED OPERATIONAL STAGES FOR A 200-m Dive With ThreE Yos

\begin{tabular}{l|cc|cc}
\hline Operational phase & \multicolumn{2}{|c}{ Clean } & \multicolumn{2}{c}{ Biofouled } \\
& $\bar{d}[\mathrm{~s}]$ & $s_{d}[\mathrm{~s}]$ & $\bar{d}[\mathrm{~s}]$ & $s_{d}[\mathrm{~s}]$ \\
\hline communications & 504.8 & 126.4 & 570.3 & 72.7 \\
actuation - surface & 86.7 & 32.7 & 85.1 & 33.6 \\
steady-state descent & 829.9 & 129.3 & 1091.0 & 230.3 \\
actuation - apogee & 148.7 & 11.6 & 145.9 & 13.9 \\
steady-state ascent & 1317.7 & 110.6 & 2000.4 & 295.6 \\
\hline total & $7,653.7$ & 978.7 & $10,537.4$ & $1,792.8$ \\
\hline
\end{tabular}

between the independent and continuous dependent variables and the mean-square errors to be normally distributed [32].

Tables V and VI show the mean and standard deviation of the power consumption and duration of the five identified operational stages, respectively. The corresponding energy consumption is displayed in Fig. 13 over a dive with three yos for a maximum depth of $200 \mathrm{~m}$.

For Seagliders, the VBD is known to contribute to $70 \%$, the computer to $15 \%$, the sensors to $7 \%$, and the telemetry to $7 \%$ of the total energy expenditure over a whole deployment [39]. Hence, the energy share of each operational stage

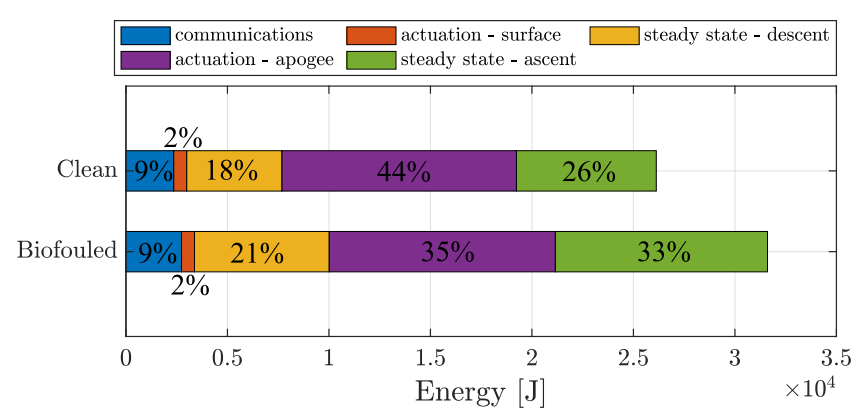

Fig. 13. Energy consumption during the identified five operational stages over a dive cycle with three yos for a 200-m dive for the clean and biofouled UG.

TABLE VII

Mean and Standard DeVIation of THE COT and Horizontal Velocity FOR A 200-m Dive With THREE YoS

\begin{tabular}{lc|cc}
\hline Variable & Units & Clean & Biofouled \\
\hline$\overline{\mathrm{COT}}$ & {$\left[\mathrm{J} \mathrm{m}^{-1} \mathrm{~kg}^{-1}\right]$} & 0.199 & 0.325 \\
$s_{\mathrm{COT}}$ & {$\left[\mathrm{J} \mathrm{m}^{-1} \mathrm{~kg}^{-1}\right]$} & 0.104 & 0.195 \\
$\dot{\dot{x}}$ & {$\left[\mathrm{~m} \mathrm{~s}^{-1}\right]$} & 0.344 & 0.185 \\
$s_{\dot{x}}$ & {$\left[\mathrm{~m} \mathrm{~s}^{-1}\right]$} & 0.129 & 0.067 \\
\hline
\end{tabular}

seems sensible. It is interesting to note that the pressure lower than atmospheric inside the pressure hull greatly helps reduce the actuation energy costs of the UG, since the VBD bladder can be retracted before descents without operating the VBD pump, which is the greatest power draw.

As can be seen in Fig. 13, the main impact that marine growth has on energy consumption is that the share of the energy consumption during steady-state descents and ascents increases from $26 \%$ and $18 \%$, respectively, to $33 \%$ and $21 \%$, respectively. As can be seen in Tables V and VI, this is mainly due to the increase in duration of these stages with marine growth due to the rise in drag and drop in speed, while the rise in power consumption is minimal.

4) Cost of Transport: Similar to that in [40], the energetic COT is quantified as the ratio of the energy expenditure and the product of the vehicle's mass and distance travelled. Table VII displays the mean and standard deviation of the COT and horizontal velocity for the clean and biofouled UG in dives with a maximum depth of $200 \mathrm{~m}$ and three yos. The distance travelled during the dive is computed from the latitude and longitude at the start and end of the dive, when the UG receives a GPS fix. Similarly, the dive duration is computed from the difference of the respective time stamps. The results from the initial five set-up dives are ignored.

As can be seen in Table VII, severe biofouling levels result in a rise in the COT of $63.3 \%$ and a drop in horizontal speed of $46.3 \%$. The COT results are comparable to that in [40], although the higher values are likely to be due to the full VBD settings used during the current deployment.

5) Rudder Angle Analysis: The Lilliefors test $(p \ll 1 \%)$ shows that the rudder angle is not normally distributed for either sets (with the clean and biofouled hull). Levene's test shows that the two sets have a significantly different variance $(p \ll 1 \%)$. 
TABLE VIII

MeAn AND STANDARd DEVIATION OF THE RUdDER ANGLE DURING STEADY-STATE CONDITIONS

\begin{tabular}{l|cc}
\hline & Clean & Biofouled \\
\hline$\overline{\delta_{\mathrm{r}}}\left[^{\circ}\right]$ & -5.846 & -5.288 \\
$s_{\delta_{\mathrm{r}}}\left[{ }^{\circ}\right]$ & 3.967 & 6.283 \\
\hline
\end{tabular}

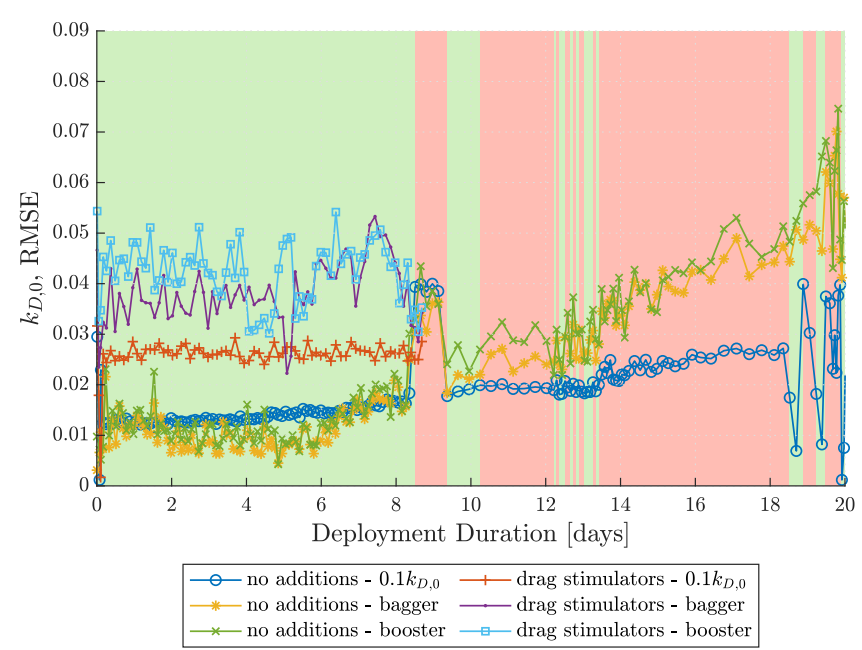

Fig. 14. Variation with time of the drag coefficient and RMSE of the boosted and bagged ensemble predictors for each dive for the two deployments. The response of the clustering algorithm is shown with green and red patches for low and high levels of marine growth, respectively.

This is also clear from Table VIII, which shows the mean and standard deviation of the rudder angle during steady-state conditions. The mean value is slightly offset from $\delta_{\mathrm{r}}=0^{\circ}$ for both data sets. Marine growth causes noticeably greater variance for the rudder angle due to the loss in rudder's effectiveness owing to the lower UGs speed in the water.

\section{B. Marine Growth Detection}

Fig. 14 shows the performance of the dynamic model and the ensembles of boosted and bagged regression trees (referred to as booster and bagger, respectively) in identifying marine growth. While the tracked variable is the RMSE for the machine learning methods, the drag coefficient is the value of interest of the dynamic model. For all methods, the difference in the tracked variable between the hull with no additions and the run with drag stimulators decreases with deployment duration due to the onset of actual biofouling.

From a closer inspection of Fig. 14, it is clear that the drag coefficient presents significant oscillations between 8 and 10 days after the start of the mission and for the last 2 days of the longer deployment (with no additions to the hull). This is reflected in a similar behavior of the predicted net buoyancy offset in Fig. 15. The serious oscillations correspond to dives where the data were stored only for descents. Hence, both modelbased and data-driven detection methods are sensitive to the symmetry of the dive data for a correct centering. Although the RMSE of the machine learning strategies is also negatively

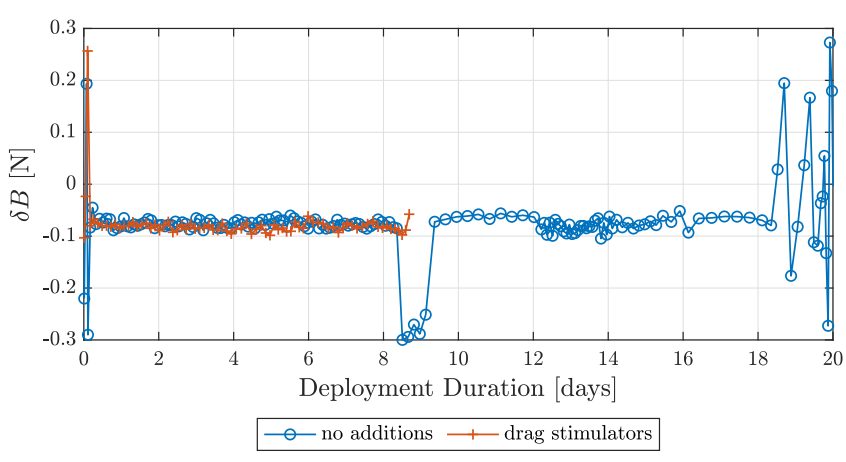

Fig. 15. Variation with time of the change in buoyancy predicted by the steadystate dynamic model for each dive for the two deployments.

influenced for these data points, the random forest and LSBoost are less affected by having data for descents only. It would be possible to reduce the error of the global optimizer used for the system identification by reducing the number of variables to one, the drag coefficient, while leaving the buoyancy offset, lift, and induced drag coefficients constant. However, as shown in [18], the buoyancy offset is fundamental in tracking wing loss and so it should be kept as a variable to be optimized.

Focusing on the machine learning methods in Fig. 14, it is clear from the steep rise in the slope of the RMSE after the six days mark for the deployment with no additions (i.e., the end of the training set) that both strategies suffer from some overfitting. The random forest seems to perform slightly better than LSBoost for both deployments, with its RMSE curve presenting less variance.

The RMSE of the ensembles of bagged and boosted regression trees and the drag coefficient are used as input to the KNN clustering algorithm after standardization with the training set data (UG with a clean hull and drag stimulators). As can be seen in Fig. 16, two natural, fully separated clusters are formed from the data of the clean and biofouled hulls. For this reason, the KNN classifier return $100 \%$ accuracy on the training and test sets and zero cross-validation error for the data with drag stimulators and the perfectly clean hull. Conversely, some dives for the whole deployment of the UG with no additions represent outliers on the plot. These are prevalently dives that are in strong currents or for which only data from the descents is available. Hence, during testing, the developed marine growth detection and identification tool makes classification errors for these dives, as shown in Fig. 14 by the green and red background patches. Nevertheless, the tool can provide guidance to the UG pilots in identifying high levels of marine growth and, thus, make a decision on whether a mission change may be needed. Tracking the curves of the drag coefficient and RMSE of the ensemble of bagged trees and of the ensemble of boosted directly may provide additional help. From an operational perspective, it will be interesting to differentiate between no or low levels of biofouling, the onset of marine growth, and high levels of biofouling in the future.

The clearly defined clusters in Fig. 16 indicate that thresholds would work well to differentiate between clean and biofouled 


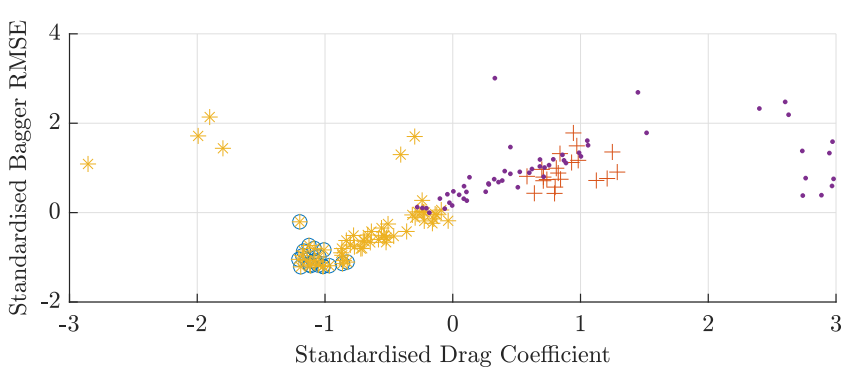

(a)

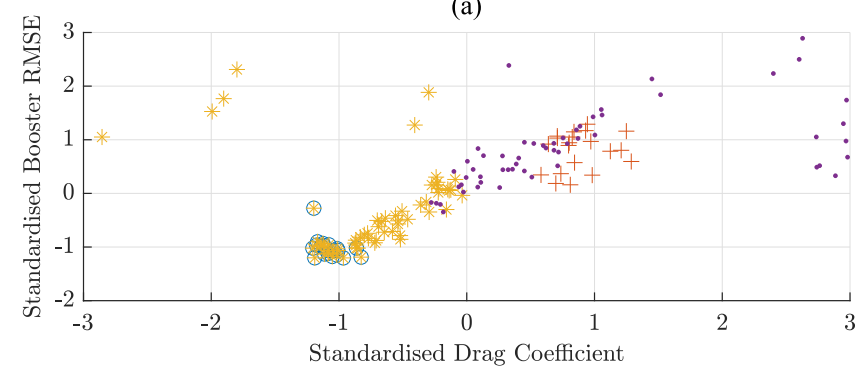

(b)

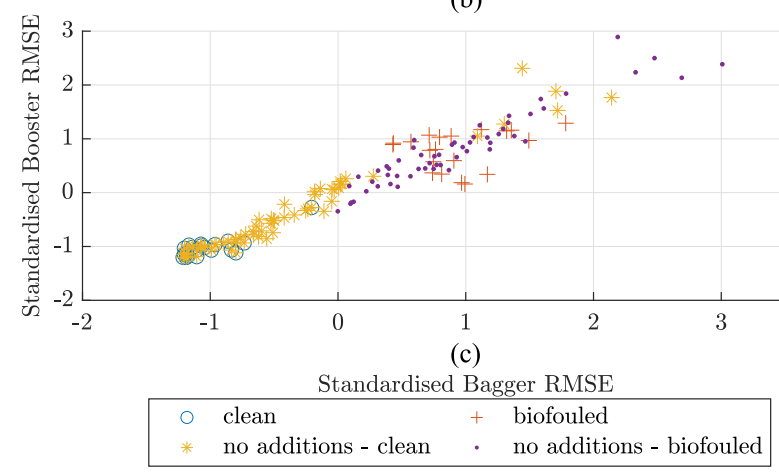

Fig. 16. Clusters identified from the standardized bagger RMSE, booster RMSE, and drag coefficient.

conditions. However, the proposed clustering procedure is more general, as new data can be integrated to update the classifier's response without having to redesign the rule-based threshold every time. Furthermore, the use of multiple input features can provide more flexibility in generalizing to different situations and vehicle types. Additionally, it is clear that the two ensemble methods present very similar performance, with their RMSE values presenting an almost linear relationship in Fig. 16. Therefore, the classifier could include the RMSE from only one method in the future in addition to the drag coefficient, in particular, the ensemble of bagged regression trees, which presents lower RMSE variance.

For each dive, the optimization of the model-based method has a computational cost in the order of $10 \mathrm{~s}$ on a modern laptop, while the ensemble of regression trees and the KNN clustering algorithm have a computational cost in the order of $1 \mathrm{~s}$ once trained. Hence, the marine growth detection system can be applied in real time after each glider's surfacing once the decimated data for the new dive are sent to the shore-based monitoring center. If excessive levels of marine growth are detected, the mission plan can be modified before the commands for the new dive are submitted. The parameters of the ensemble of regression trees can be updated offline while the vehicle is performing a new dive.
The normalization of the drag coefficient and the RMSE of the ensemble of bagged regression trees are fundamental to generalize the response of the clustering algorithm to other Slocum UGs. In fact, the method is likely to be reliable only for Slocum G2 vehicles with no large appendages, e.g., Ocean Microstructure Gliders (OMG), which have a much higher initial drag coefficient and, thus, will experience a much lower relative increase in drag due to marine growth. Hence, new data are needed specific to those vehicles. Furthermore, the selected $\mathrm{KNN}$ algorithm is suitable only if used with a small reference data set as in this case. If a large data set is used, i.e., from many missions where the UG suffered from severe biofouling, then parametric solutions will be better than nonparametric methods, e.g., KNN, because nonparametric schemes need to use the whole data set at deployment time. If much larger data sets are available, e.g., from multiple deployments of UGs with similar scientific packages, clean hulls and no faults, then the machine learning solution should also be replaced with a deep learning strategy, which is more scalable [21].

\section{CONCLUSION}

High levels of marine growth, as simulated by the drag stimulators, on the UGs performance were observed to cause a drop in vertical velocity for the same VBD volume due to an increase of $92 \%$ of the drag coefficient and $37 \%$ of the induced drag coefficient. Furthermore, the share of steady-state descents and ascents increased from $26 \%$ and $18 \%$ to $33 \%$ and $21 \%$, respectively, of the overall total energy consumption for a typical three-yo dive. This is caused by the longer duration of these stages due to the lower vertical speed and a significantly greater use of the actuators, as the rudder becomes less effective due to the smaller lift force generated by the lower energy flow. As a result, high levels of marine growth will significantly reduce deployment duration, with pilots needing to replan the mission if high levels of biofouling are detected.

The condition monitoring system presented here is based on the blending of the estimated drag coefficient and RMSE of the vertical velocity predicted by an ensemble of bagged regression trees and an ensemble of boosted regression trees. It is able to successfully detect and identify the onset of high levels of marine growth for each dive. A small fraction of classification error occurs for some outlier data points due to dives with strong marine currents or data stored only for descents. The dynamic model is particularly affected by having data only for descents, so that navigation data from both descents and ascents should be sent by satellite after decimation if model-based diagnostics are to be used. The scientific data are sufficient for either descents or ascents, since these can be interpolated for the remaining portion of the dive. Although very challenging in practice, future work could include the detection from low to severe levels and different types of biofouling.

Thanks to the normalization of the drag coefficient and the RMSE error the strategies trained with the data set collected during these field tests can be generalized to other gliders. However, a new training data set is likely to be required for different types of UGs or vehicles with large extrusions, e.g., OMG Slocum UGs. 
The early detection of the onset of marine growth will enable pilots to take recovery actions, e.g., reducing the time the gliders spend near the surface, as well as replan the deployment considering the quantified impact of biofouling on power consumption.

\section{ACKNOWLEDGMENT}

The authors would like to thank Stephen C. A. Woodward at Marine Autonomous and Robotic Systems National Oceanography Centre Southampton, U.K., for his help with developing the drag stimulators used during the field tests. The authors would also like to thank Rayco Moran, Patricia Rivera Rodriguez, and Eduardo Caudet for their support during the deployments.

\section{REFERENCES}

[1] D. L. Rudnick, "Ocean research enabled by underwater gliders," Аnnu. Rev. Mar. Sci., vol. 8, no. 1, pp. 519-541, 2016.

[2] C. D. I. Haldeman, D. K. Aragon, T. Miles, S. M. Glenn, and A. G. Ramos, "Lessening biofouling on long-duration AUV flights: Behavior modifications and lessons learned," in Proc. MTS/IEEE OCEANS Conf., 2016, pp. 1-8.

[3] M. Eichhorn, D. Aragon, Y. A. Shardt, and H. Roarty, "Modeling for the performance of navigation, control and data post-processing of underwater gliders," Appl. Ocean Res., vol. 101, Aug. 2020, Art. no. 102191.

[4] C. Deutsch, J. Kuttenkeuler, and T. Melin, "Glider performance analysis and intermediate-fidelity modelling of underwater vehicles," Ocean Eng., vol. 210, Aug. 2020, Art. no. 107567.

[5] K. Hamilton, D. M. Lane, K. E. Brown, J. Evans, and N. K. Taylor, "An integrated diagnostic architecture for autonomous underwater vehicles," J. Field Robot., vol. 24, no. 6, pp. 497-526, Jun. 2007.

[6] Y. Ran, X. Zhou, P. Lin, Y. Wen, and R. Deng, "A survey of predictive maintenance: Systems, purposes and approaches," IEEE Syst. J., early access, May 6, 2019. doi: 10.1109/JSYST.2019.2905565.

[7] X. Hong, R. J. Mitchell, S. Chen, C. J. Harris, K. Li, and G. W. Irwin, "Model selection approaches for non-linear system identification: A review," Int. J. Syst. Sci., vol. 39, no. 10, pp. 925-946, Oct. 2008.

[8] S. R. Saufi, Z. A. B. Ahmad, M. S. Leong, and M. H. Lim, "Challenges and opportunities of deep learning models for machinery fault detection and diagnosis: A review," IEEE Access, vol. 7, pp. 122 644-122662, 2019.

[9] A. Stetco et al., "Machine learning methods for wind turbine condition monitoring: A review," Renewable Energy, vol. 133, pp. 620-635, Apr. 2019.

[10] A. Freddi, S. Longhi, and A. Monteriù, "Actuator fault detection system for a remotely operated vehicle," IFAC-PapersOnline, vol. 46, no. 33, pt. 1, pp. 356-361, 2013.

[11] Y. Wang and M. Zhang, "Research on test-platform and condition monitoring method for AUV," in Proc. IEEE Int. Conf. Mechatron. Autom., 2006, pp. 1673-1678.

[12] Y. S. Sun, X. R. Ran, Y. M. Li, G. C. Zhang, and Y. H. Zhang, "Thruster fault diagnosis method based on Gaussian particle filter for autonomous underwater vehicles," Int. J. Nav. Archit. Ocean Eng., vol. 8, no. 3, pp. 243-251, May 2016.

[13] F. Yao, F. Wang, and M. Zhang, "Weak thruster fault detection for autonomous underwater vehicle based on artificial immune and signal pre-processing," Adv. Mech. Eng., vol. 10, no. 2, Feb. 2018.

[14] W. R. Harris, "Anomaly detection methods for unmanned underwater vehicle signature redacted signature redacted signature redacted," M.Sc thesis, Sloan Schl. Manage., Massachusetts Inst. Technol., Cambridge, MA, USA, 2015.

[15] B. Y. Raanan et al., "A real-time vertical plane flight anomaly detection system for a long range autonomous underwater vehicle," in Proc. MTS/IEEE OCEANS Conf., 2015, pp. 1-6.

[16] B.-Y. Raanan et al., "Automatic fault diagnosis for autonomous underwater vehicles using online topic models," in Proc. MTS/IEEE OCEANS Conf., 2016, pp. 1-6.

[17] B. Y. Raanan et al., "Detection of unanticipated faults for autonomous underwater vehicles using online topic models," J. Field Robot., vol. 35, no. 5, pp. 705-716, Aug. 2018.

[18] E. Anderlini, C. A. Harris, G. Salavasidis, A. Lorenzo, A. B. Phillips, and G. Thomas, "Autonomous detection of the loss of a wing for underwater gliders," in Proc. IEEE/OES Autonom. Underwater Veh. Symp., 2020, pp. 1-6.
[19] E. Anderlini et al., "Identification of the dynamics of biofouled underwater gliders," in Proc. IEEE/OES Autonom. Underwater Veh. Symp., 2020, pp. $1-6$.

[20] ITTC, "Recommended procedures and guidelines - ship models," 7.5-0101-0, pp. 4-5, 2011.

[21] I. Goodfellow, Y. Bengio, and A. Courville, Deep Learning. Cambridge, MA, USA: MIT Press, 2016.

[22] C. Aldrich and L. Auret, "Fault detection and diagnosis with random forest feature extraction and variable importance methods," IFAC-PapersOnline, vol. 43, no. 9, pt. 1, 2010, pp. 79-86.

[23] D. C. Webb, P. J. Simonetti, and C. P. Jones, "SLOCUM: An underwater glider propelled by environmental energy," IEEE J. Ocean. Eng., vol. 26, no. 4, pp. 447-452, Oct. 2001.

[24] O. Schofield et al., "Slocum gliders: Robust and ready," J. Field Robot., vol. 24, no. 6, pp. 474-485, 2007.

[25] "Slocum G2 glider operators manual," Teledyne Webb Research, North Falmouth, MA, USA, Tech. Rep. P/N 4343, Rev. B, 2012. [Online]. Available: www.webbresearch.com

[26] D. M. Vogt et al., "Shipboard design and fabrication of custom 3D-printed soft robotic manipulators for the investigation of delicate deep-sea organisms," PLoS ONE, vol. 13, no. 8, Aug. 2018, Art. no. e0200386.

[27] L. Merckelbach, D. Smeed, and G. Griffiths, "Vertical water velocities from underwater gliders," J. Atmos. Ocean. Technol., vol. 27, no. 3, pp. 547-563, 2010.

[28] L. Merckelbach, A. Berger, G. Krahmann, M. Dengler, and J. R. Carpenter, "A dynamic flight model for Slocum gliders and implications for turbulence microstructure measurements," J. Atmos. Ocean. Technol., vol. 36, pp. 281-296, Jan. 2019.

[29] T. McDougall and P. Barker, "Getting started with TEOS-10 and the Gibbs seawater (GSW) oceanographic toolbox," SCOR/IAPSO WG 127, pp. 1-28, 2011.

[30] Z. Ugray, L. Lasdon, J. Plummer, and F. Glover, "Scatter search and local NLP solvers : A multistart framework for global optimization," Inf. Syst., vol. 19 , no. 3, pp. 328-340, 2007.

[31] R. H. Byrd, J. C. Gilbert, and J. Nocedal, "A trust region method based on interior point techniques for nonlinear programming," Math. Program., B, vol. 89, no. 1, pp. 149-185, 2000.

[32] T. Hastie, R. Tibshirani, and J. Friedman, Elements of Statistical Learning Data, 2nd ed. Berlin, Germany: Springer-Verlag, 2009.

[33] C. A. Harris et al., "Oceanids C2: An integrated command, control, and data infrastructure for the over-the-horizon operation of marine autonomous systems," Front. Mar. Sci., vol. 7, Jun. 2020.

[34] K. E. Knutsen, G. Manno, and B. J. Vartdal, "Beyond condition monitoring in the maritime industry," DNV-GL, Tech. Rep., 6-2014, 2014.

[35] K. P. Murphy, Machine Learning: A Probabilistic Perspective, 1st ed. Cambridge, MA, USA: MIT Press, 2012.

[36] M. Krzywinksi and N. Altman, "Classification and regression trees," Nature Methods, vol. 14, no. 8, pp. 757-758, 2017.

[37] T. G. Dietterich, "Ensemble methods in machine learning," in Proc. 1st Int. Workshop Multiple Classifier Syst., 2000, pp. 1-15.

[38] T. Hastie, R. Tibshirani, and J. Friedman, The Elements of Statistical Learning: Data Mining, Inference, and Prediction, 2nd ed. Berlin, Germany: Springer, 2009.

[39] IRobot, "1KA Seaglider user's guide," Tech. Rep. 4220126, Rev. C, Jan. 2012.

[40] A. B. Phillips et al., "Nature in engineering for monitoring the oceans: Comparison of the energetic costs of marine animals and AUVs," in Further Advances in Unmanned Marine Vehicles (IET Control Eng. Ser.), G. Roberts and R. Sutton, Eds. London, U.K.: IET, 2012, pp. 373-405.

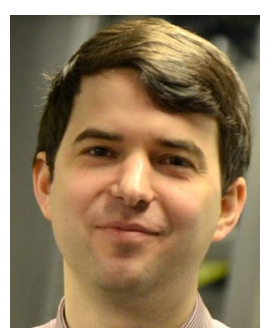

Enrico Anderlini received the M.Eng. (Hons.) degree in ship science from the University of Southampton, Southampton, U.K., in 2013, and the Eng.D. degree in offshore renewable energy from IDCORE, a partnership of the Universities of Edinburgh, Exeter, and Strathclyde, U.K., in 2018.

Since 2017, he has been a Researcher with the Marine Research Group, University College London, London, U.K., where he has been a Senior Research Fellow since 2019. His research interests include fault-tolerant control for marine autonomous systems and offshore renewable energy devices. 


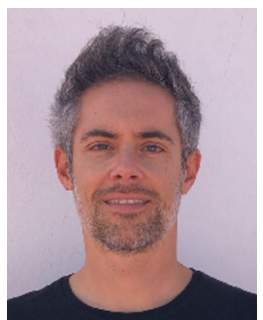

Daniel Alcaraz Real-Arce received the M.S. degree in renewable energies from the University of San Pablo CEU Madrid, Madrid, Spain, in 2010, and the M.S. degree in telecommunication engineering and the Ph.D. degree in telecommunication technologies from the University of Las Palmas de Gran Canaria (IUMA), Las Palmas de Gran Canaria, Spain, in 2011 and 2014, respectively.

He has been an H2020 Project Manager with Oceanic Platform of the Canary Islands, Las Palmas, Spain, for the past six years, managing projects related to ocean observation, autonomous marine vehicles, robotics, educational activities, and dissemination initiatives into international teams. He has previously worked managing R\&D projects with the Institute of Applied Microelectronics, IUMA, and as a private consultant.

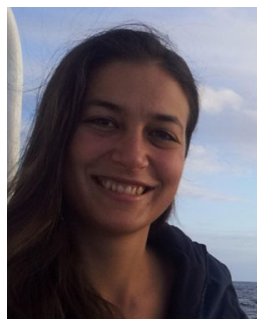

Tania Morales received the Engineering degree in telecommunications from the University of Las Palmas in Gran Canaria, Las Palmas de Gran Canaria, Spain, in 2011, and the master's degree in telematics engineering from Vigo University, Vigo, Spain, in 2015.

Since 2011, she has been a part of the glider's team with Oceanic Platform of the Canary Islands (PLOCAN), Las Palmas, Spain, having been involved in more than 40 missions carried out with marine autonomous vehicles. She has also been in charge of the design and implementation of PLOCAN data management IT infrastructure for both mobile and fixed ocean observation systems. Since 2015, she has been the Project Manager for EU projects related to underwater robotics and ocean data management.

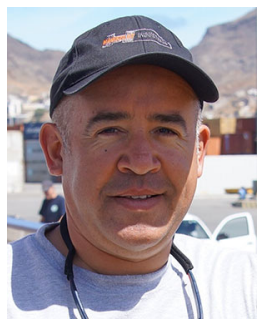

Carlos Barrera received the degree in oceanography from the University of Las Palmas de Gran Canaria (ULPGC), Spain, in 1998.

He has been the Head of the Ocean-Vehicles Unit with Oceanic Platform of the Canary Islands (PLOCAN), Las Palmas, Spain, since 2007. He is an Oceanographer. He has more than 25 years of experience in marine technology with a focus on autonomous ocean-observing platforms. His technical and operational tasks are time shared with desk activities related to $R \& D$ project-coordination, ocean governance initiatives, and services management.

Mr. Barrera is currently a Chair of the EuroGOOS Ocean-Glider Task Team.

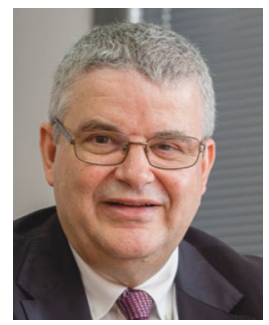

José Joaquín Hernández-Brito received the Ph.D. degree in chemistry from the University of La Laguna, San Cristóbal de La Laguna, Spain, in 1986.

He has been a Senior Lecturer with the Faculty of Marine Sciences, University of Las Palmas in Gran Canaria, Las Palmas de Gran Canaria, Spain, since 1983. He has held several R\&D management positions such as the Dean of the Faculty of Marine Science, Director of the Research Results Transfer Office (TTO), Director of the Scientific and Technological Park, research Vice-Rector, Manager of Innovation with the Canary Islands Institute of Technology, and Director of the Marine Institute of the Canary Islands. He was with the Oceanic Platform of the Canary Islands (PLOCAN), Las Palmas, Spain, from 2009 to 2019, as a Manager and the Science, Technology and Innovation Director. In 2019, he was appointed CEO with the AIR CENTRE, a position he served until he was appointed as the CEO with PLOCAN in January 2020.

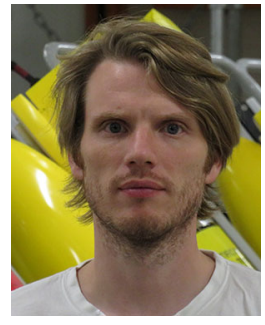

Alexander B. Phillips received the M.Eng. (first class Hons.) degree in ship science and the Ph.D. degree in marine hydrodynamics from the University of Southampton, Southampton, U.K., in 2004 and 2010, respectively.

From 2010 to 2015, he was a Postdoctoral Researcher with the Marine Robotics Laboratory, University of Southampton. In 2015, he joined the National Oceanography Centre, Southampton, as the Head of Marine Autonomous Systems Development. In 2018, he became the Head of the Marine Autonomous and Robotic Systems Development Group, where he is responsible for the development of a range of new robotic platforms for ocean science applications.

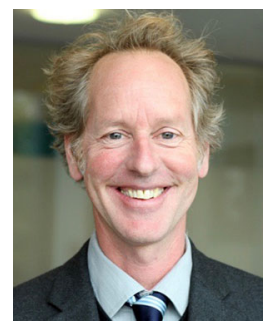

Giles Thomas received the B.Eng. (Hons.) degree in naval architecture and shipbuilding and the M.Phil. degree in naval architecture and the Ph.D. degree in engineering from the University of Newcastle upon Tyne, Newcastle upon Tyne, U.K., in 1988 and 1991, respectively, and the Ph.D. degree from the University of Tasmania, Hobart, TAS, Australia, in 2003.

$\mathrm{He}$ is currently the BMT Chair of Maritime Engineering with University College London, London, U.K.

Dr. Thomas is a Fellow of the Royal Institution of Naval Architects and has more than 30 years of research and development experience in the fields of hydrodynamics and fluid-structure interaction working with a large range of industry partners. 